\title{
Meeting report: 32nd international mammalian genome conference
}

\author{
Jacob Moskowitz ${ }^{1} \cdot$ Lauren Tracey $^{2,3} \cdot$ Samuel Widmayer $^{4} \cdot$ Beth Dumont $^{5}$ (i)
}

Received: 6 February 2019 / Accepted: 27 March 2019 / Published online: 1 April 2019

(c) Springer Science+Business Media, LLC, part of Springer Nature 2019

\begin{abstract}
Over 150 scientists from more than 50 research institutions and eight countries attended the 32 nd annual meeting of the International Mammalian Genome Society (IMGS) held in Rio Mar, Puerto Rico. Attendees included predoctoral and postdoctoral trainees, junior investigators, clinicians, industry professionals, and established leaders in mammalian genetics and genomics. From November 11-14, major scientific advances in the fields of systems genetics, developmental biology, cancer, human disease modeling, and bioinformatics were showcased in a series of 66 poster and 54 platform presentations. Here we provide an overview of the meeting's proceedings and summarize the exciting, novel research findings communicated by conference participants that, collectively, are advancing the frontiers of mammalian genetics and genomics.
\end{abstract}

\section{Introduction}

Scientists from across the globe converged on the sandy beaches of Puerto Rico for the 32nd International Mammalian Genome Conference (IMGC), held at the Wyndham Grand in Rio Mar from the November 11-14, 2018. The International Mammalian Genome Society's (IMGS) annual conference brought over 150 participants from around the

Lauren Tracey and Samuel Widmayer have contributed equally to manuscript.

Jacob Moskowitz

moskowitzj@missouri.edu

$\triangle$ Beth Dumont

beth.dumont@jax.org

Lauren Tracey

lauren.tracey@sickkids.ca

Samuel Widmayer

sjwidmay@ncsu.edu

1 Department of Veterinary Pathobiology, University of Missouri, 4011 Discovery Drive, Columbia, MO 65201, USA

2 Department of Molecular Genetics, University of Toronto, Toronto, ON, Canada

3 Genetics and Genome Biology Program, The Hospital for Sick Children, Toronto, ON, Canada

4 Graduate Program in Genetics, Department of Biological Sciences, North Carolina State University, Raleigh, NC, USA

5 The Jackson Laboratory, 600 Main Street, Bar Harbor, ME 04609, USA world, building on the society's successful IMGC2017 held in conjunction with the Mouse Molecular Genetics (MMG) group at the European Molecular Biology Laboratory (EMBL) in Heidelberg, Germany (Sanchez-Andrade et al. 2018). For the duration of IMGC2018, a highly diverse group of scientists including new trainees and long-standing members of the community shared exciting new advances in a wide range of topics, reflecting the expansive scope of professionals drawn to IMGS. This report serves to update the wider research community on the topics covered throughout the IMGC2018 meeting in Puerto Rico.

The 32nd IMGC commenced with a series of expert-led, focused workshops open to conference participants interested in gaining new skills and knowledge (Table 1). The main conference consisted of 11 sessions of oral presentations given by invited and selected presenters covering a broad scope of topics applicable to the wider genetics community including: (1) Development, Epigenetics, and Stem cells; (2) Comparative, Evolutionary, and Computational Genomics; (3) Animal Models of Human Disease; (4) Translational and Systems Genetics; (5) Resources and Technical Advances. Additionally, IMGC2018 featured three plenary lectures and two poster sessions with a total of 66 posters presented. This stimulating 4-day scientific program concluded with dinner and social activities at the Hacienda Siesta Alegre, a stunning outdoor venue perched on a picturesque mountainside with breathtaking views of the Puerto Rican rainforest. Abstracts from the meeting can be found under the History tab at http://www.imgs.org. 
Table 1 International mammalian genome conference 2018 workshops

\begin{tabular}{lll}
\hline Topic & Facilitator & Home Institution \\
\hline CRISPR & Channabasavaiah Gurumurthy & University of Nebraska \\
Ensembl & Toby Hunt & European Bioinformatics Institute \\
GeneWeaver & Jason Bubier & The Jackson Laboratory \\
IMPC & Ann-Marie Mallon and Anna Swan & Medical Research Council Harwell Institute \\
Next generation & Thomas Keane & European Bioinformatics Institute \\
sequencing & & \\
\hline
\end{tabular}

\section{Trainee symposium}

The IMGS has a long-standing commitment to advocating new scientific talent, demonstrated by the participation of 46 graduate and postdoctoral trainees of whom 30 were awarded trainee scholarships for conference travel. Trainee attendees had the opportunity to interact with scientists in the community during a mentor-mentee lunch, which prompted engaging discussions on diverse topics relevant to trainee development. The trainee experience at IMGC was highlighted by the trainee symposium. Sixteen graduate and postdoctoral researchers presented their work to the community in this stimulating and encouraging environment. Among multiple awards given to trainee presenters throughout the conference (Table 2), five presenters in the trainee symposium were selected to receive the Lorraine Flaherty Outstanding Oral Presentation Award. This prestigious honor provided awardees the opportunity to present their research in the main conference sessions.

Exceptional trainee presentations were given that addressed central questions in the field of stem cell biology. Candice Byers (The Jackson Laboratory, USA) presented her work dissecting the genetic architecture of stem cell differentiation using mouse embryonic stem cells (mESCs) derived from 33 BXD lines. Byers maintained these cells in ground state pluripotency conditions and identified QTLs for chromatin accessibility (caQTLs) and gene expression (eQTLs). Several trans-QTLs were identified for both genomic traits, implicating a common regulatory system for chromatin state and gene expression. These shared QTLs were enriched for KRAB zinc finger proteins (KRAB-ZFPs), which are known to regulate expression of transposable elements (TEs). Byers showed that TEs were often differentially expressed between the C57BL/6J and DBA/2J founders and that the regulation of TE expression is controlled by caQTLs implicated in this work. Her findings raise the intriguing possibility that TE and KRAB-ZFPs are engaged in an evolutionary conflict over the control of gene expression networks in mESCs during differentiation. Joseph Palmer (University of Alabama-Birmingham, USA) identified genomic signatures associated with stem cell quiescence using a melanocyte stem cell model. Employing RNA sequencing from stem cells at three different ages, he was able to identify core regulatory networks associated with both quiescence and activation. Interestingly, he also identified a subtype of deep quiescent cells that remain quiescent in advancing age. Genes that are differentially expressed between cells at different levels of quiescence provide intriguing candidates to pursue as key determinants of maintenance of proper stem cell function in aging cells.

Two trainee presentations focused on the genetic dissection of Factor 5 Leiden (F5L), a risk factor for venous thromboembolism (VTE). Marisa Brake (Oakland University, USA) presented an extensive dissection of an ENU screen for dominant suppressors of VTE in the $F 5^{t m 2 D g i} / F 5^{t m 2 D g i}$ $T f p i^{t m l G j b} /+$ mouse line, which yields perinatal lethality. Linkage analysis implicated $F 3$ as a potential candidate in one thrombosuppressor line, though no coding mutations could be recovered. Brake then performed whole-genome sequencing on five mice from one thrombosuppressor line and identified 23 non-coding variants within the linkage peak. Amy Siebert (Oakland University) presented work from alternate thrombosuppressor lines resulting from the same ENU screen. In these lines, a R258G missense mutation in the Actr2 gene was revealed as a candidate modifier of lethality. These observations led Siebert to use CRISPRCas9 genome editing to create knockout alleles of the Actr2 mutation. Surprisingly, offspring carrying the knockout or missense allele could not be obtained, confirming that both Actr2 alleles are required for survival.

Two trainee talks focused on "dark matter" regions of the mammalian genome that continue to elude full characterization on account of their repetitive architecture: centromeres and transposable elements (TEs). Centromeres are essential for genome stability and cellular mechanics, but paradoxically, are rapidly evolving at the sequence level. In order to better understand the diversity and evolution of these genomic regions, Uma Arora (The Jackson Laboratory) introduced a pipeline to query $k$-mer frequencies from unmapped sequencing reads in diverse mouse strain genomes. Her preliminary findings uncovered significant variation in the frequency of k-mers that comprise the consensus centromere satellite sequence, suggesting remarkable diversity in copy number at these critical genomic loci. TE 


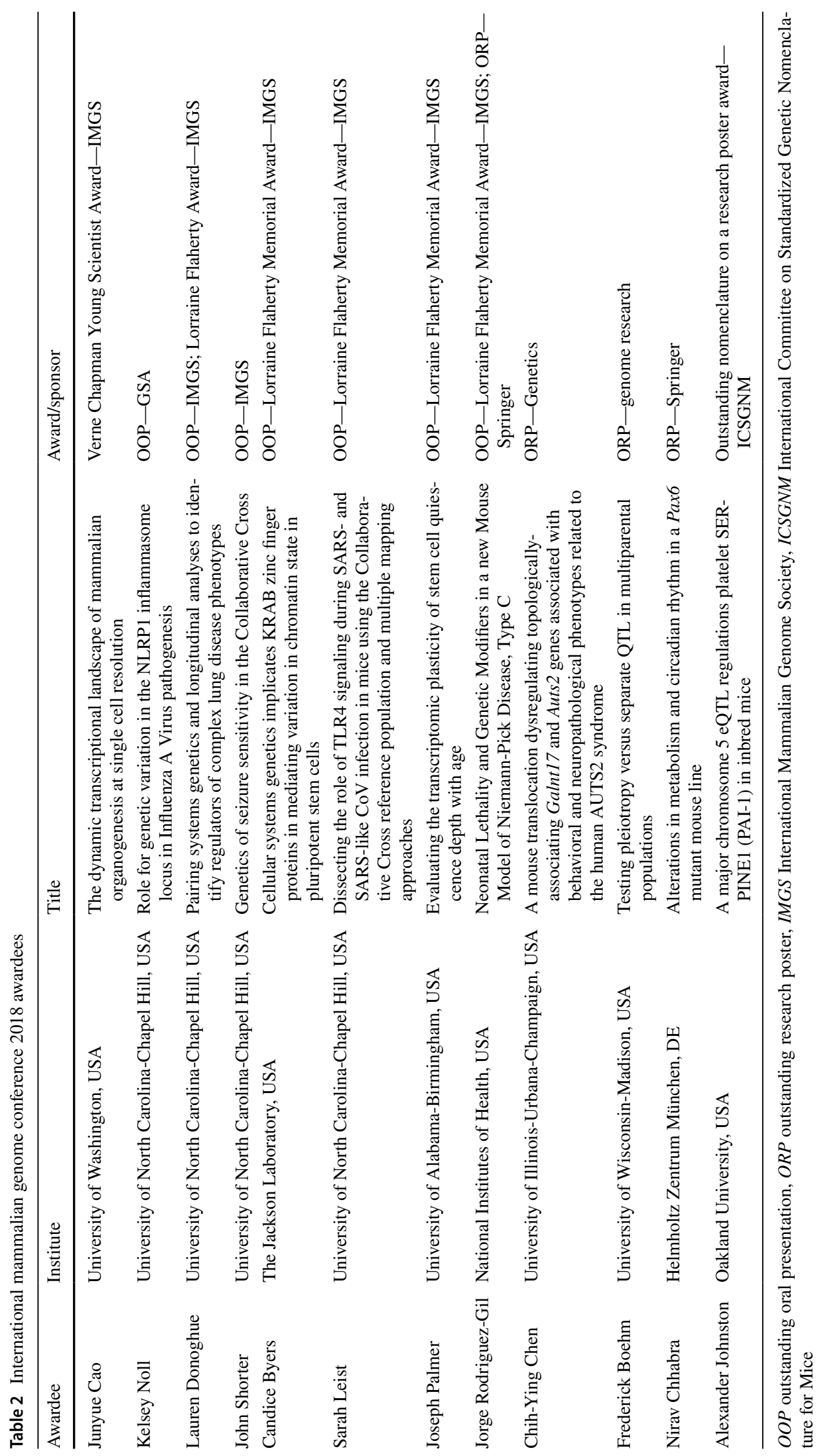


insertion is an important source of phenotypic variability and genomic instability, yet little is known about the distribution or functional impact of TEs in diverse genetic contexts. Anwica Kashfeen (UNC-Chapel Hill, USA) presented the ELITE algorithm for high-throughput identification of TEs across multiple mouse populations. This method has high sensitivity and remarkable accuracy, underscoring its promise for the discovery and functional validation of TE insertions across current and future genomic resources for genetically diverse mice.

Two trainee talks underscored the power of mouse models for toxicology research and drug testing. Lauren Lewis (Texas A\&M University, USA) exposed 50 recombinant inbred Collaborative Cross (CC) strains to 1,3-butadiene (BD), a known human carcinogen, to address whether the genotoxic effects of exposure were heritable. THB-Gua adduct levels and several histone modifications showed strain-specific responses to BD. Furthermore, she observed an inverse relationship between the level of THB-Gua adducts after exposure and $\mathrm{H} 3 \mathrm{~K} 27 \mathrm{ac} / \mathrm{H} 327 \mathrm{me} 3$ ratio prior to exposure, demonstrating that the pre-exposure epigenetic landscape may influence the genotoxicity of BD in certain CC strains. Yuna Son (Florida State University, USA) evaluated the importance of epigenetic changes such as DNA methylation and posttranslational histone modifications in differential responses to steroid treatment of keloid lesions. Her ongoing work aims to test the hypothesis that genetic and epigenetic heterogeneity alter the diverse response to wound healing and steroid treatment for keloids in humans.

Mice have also served as prominent models for dissecting the genetic basis of speciation. Prior work has established that a genetic interaction between $\operatorname{Prdm} 9$ and a locus on the $\mathrm{X}$ chromosome leads to hybrid male sterility (HMS) in crosses between two closely related house mouse subspecies, Mus musculus musculus and M. m. domesticus. Sam Widmayer (North Carolina State University, USA) presented his work using a panel of hybrid male mice that carried identical genotypes at these known HMS loci in order to uncover genetic background effects on HMS phenotypes. He showed that, not only did fertility vary across the panel, but that some genetic backgrounds exhibited age-dependent fertility. His findings demonstrate the existence of segregating modifiers of HMS among inbred mouse strains, hinting at under-appreciated levels of genetic complexity in HMS.

Several trainee talks focused on uncovering the genetic basis of diverse human diseases, reflecting a substantial focus on applying new genetic tools to further our understanding of disease pathogenesis. Lauren Donoghue (UNC-Chapel Hill) used CC mice to study genetic mechanisms underlying host responses to chronic allergen exposure. Her work mapped the Bpifbl gene as a significant regulator of mucin production, which plays an important role in host defense and contributes to hyper-secretion of mucus associated with chronic allergic exposures. Sarah Leist (UNC-Chapel Hill) used similar mapping techniques in her studies identifying determinants of genetic susceptibility to Severe Acute Respiratory Syndrome coronavirus (SARS-CoV). Her QTL analyses yielded multiple candidate genes common to the TLR4 pathway, highlighting the importance of innate immunity in the host response to some viral pathogens. Chih-Ying Chen (University of Illinois, Urbana-Champaign, USA) introduced a new model for neurological disease associated with AUTS2 syndrome. Her research aims to understand the causative role of mutations in topologically associated genes and non-coding regions in the Auts 2 domain. Chen's model demonstrates the complexity of coding and non-coding contributions to mammalian disease.

Nirav Chhabra (Helmholz Zentrum Munchen, Germany) demonstrated the pleiotropic nature of PAX6, which codes for the paired box protein 6 and has been previously implicated in glucose intolerance and diabetes. Chhabra showed that altered expression of several circadian genes in Pax6 mutant mice results in circadian rhythm defects, changes in metabolic flexibility, and abnormal feeding patterns. Jorge Jaimes-Alvarado (Texas A\&M University, USA) investigated epidermal growth factor receptor (Egfr)-independent mechanisms of colorectal cancer development. Jaimes-Alvarado used conditional knockouts of $E g f r$ in genetically susceptible $A p c^{M i n}$ mice to first demonstrate faster growth of tumors. He then used RNA-seq to identify differentially expressed genes in colonic tumors corresponding to the IL10 receptor pathway, uncovering a potential role for immunoregulation in Egfr-independent colorectal cancer.

Leandro Prado (Duke University School of Medicine, USA) and Jorge Rodriguez-Gil (National Institutes of Health, USA) both hypothesized that genetic modifiers shape variation in their model phenotypes of interest. Prado created a series of reciprocal congenic mouse strains between $\mathrm{C} 3 \mathrm{H} / \mathrm{HeJ}$ and $\mathrm{C} 57 \mathrm{BL} 6 / \mathrm{J}$ to test the allelic effects of a previously identified QTL on infarct volumes. He showed that reciprocal congenic strains exhibit variable cerebral infarct volumes and nominated Msrl as a candidate gene with strain-specific effects on infarct size. Rodriguez-Gil carried out a linkage analysis to identify marker loci associated with variable phenotypic outcomes in a mouse model of the endosomal/lysosomal cholesterol storage disease, Niemann Pick disease Type C.

Trainee-led research was also highlighted during two poster sessions and several platform presentations during the main conference sessions. All trainee presentations were evaluated by anonymous judges, and merit awards were given for outstanding oral and poster presentations (Table 2). 


\section{Development, epigenetics, and stem cells}

Several presented talks highlighted exciting new advances in our understanding of cell differentiation and fate in the context of both normal development and disease. Dan Skelly (The Jackson Laboratory) presented his work investigating genetic determinants of variable success in deriving embryonic stem cells (ESCs) from diverse inbred strains. He performed RNA-sequencing and ATACseq (Assay for Transposase-Accessible Chromatin using sequencing) on sensitized ESCs derived from Diversity Outbred (DO) mice. Using mediation analysis of hundreds of QTLs, he identified six causal regulators of ground state pluripotency. In a series of elegant allele swap experiments, he showed that a single variant within the Lifr gene drives variation in the expression of 202 genes and influences the ease of ESC derivation in DO founder strains. Jesse Raab (UNC-Chapel Hill) presented novel results expanding our knowledge of genetic drivers of cell fate. He showed that Sox 9 expression defines differentiated cell types within the intestine and carried out an integrated analysis of gene expression, chromatin accessibility, and DNA methylation to elucidate the regulatory landscape of these cell populations. Overall, his work highlighted the dynamic control of chromatin by transcription factors.

The work of both Rebekah Tillotson (Hospital for Sick Children, Canada) and J. Andrew Pospisilik (Max Planck Institute of Immunobiology and Epigenetics, Germany) highlighted the critical functions of chromatin regulators in development and disease. Tillotson studies MECP2, which binds symmetrically methylated DNA (5-mCG) as well as asymmetrically methylated DNA (5-mCAC) through its methyl-CpG-binding domain (MBD). MECP2 mutations are responsible for over 95\% of Rett Syndrome cases. Tillotson showed that MECP2's MBD is not functionally replaceable by the MBD of its family member MBD2, which can only bind 5-mCG. She found that mice with modified MECP2 containing MBD2's DNA binding domain have Rett-Syndrome-like characteristics. Interestingly, not all phenotypes recapitulate classical Rett Syndrome in the mouse, suggesting MECP2's 5-mCAC binding has unique functional properties in the context of this disease. Pospisilik presented his work on the role of chromatin regulators in susceptibility to complex diseases such as obesity and diabetes. He focused on the Polycomb Repressive Complex 2 (PRC2) epigenetic complex and demonstrated an important role in islet beta-cell identity. Pospisilik showed that dysfunctional PRC2 can lead to de-differentiation of beta cells and diabetes.

Junyue Cao (University of Washington, USA) delivered a tour-de-force presentation on the transcriptional networks that govern core developmental pathways during mouse embryogenesis. Using "whole-organism" single-cell RNA sequencing, Cao probed over 60 embryos between ages E9.5 and E13.5, surveying more than 2 million single cells. Dimensionality reduction and clustering approaches enabled him to identify over 500 distinct cell states during organogenesis. Cao leveraged novel analytical methods to transcriptionally map the trajectories of 56 cell fate transitions corresponding to the development of all major organs in the mouse. Cao was awarded the Verne Chapman Young Scientist Award for the best trainee presentation (Table 2).

\section{Comparative, evolutionary, and computational genomics}

Multiple talks in this session utilized large-scale genomic approaches to address outstanding questions in the fields of evolutionary and comparative genomics. Fernando PardoManuel de Villena (UNC-Chapel Hill) and Gary Churchill (The Jackson Laboratory) employed genomic approaches to probe the landscape of new mutations the mouse genome. Pardo-Manuel de Villena used ELITE (see Anwica Kashfeen in Trainee Session) to scan three largely independent mouse populations for TE insertions and characterize the distribution and functional consequences of over 19,500 TE insertion sites among inbred strains. TE insertion rates varied tremendously among inbred mouse strains, hinting at the presence of segregating genetic modifiers of the TE mutation rate. Over $2700 \mathrm{TE}$ insertion sites were common to multiple strains, suggesting that many TE insertions are effectively neutral. However, Pardo-Manuel de Villena also identified $\sim 400 \mathrm{TE}$ insertions that are predicted to be deleterious. These variants may represent an important source of phenotypic variation in studies of complex traits in the mouse. Churchill used whole genome sequences from a pedigreed C57BL6/J mouse family to address the extent of genetic drift in commonly used inbred mouse strains. Churchill emphasized the difficulty of characterizing true drift from sequencing errors and emphasized the need for vigilance among investigators to reduce the impact of drift on our models.

$\operatorname{Prdm} 9$ plays critical roles in the epigenetic specification of meiotic recombination hotspots and the maintenance of subspecies barriers between M. m. musculus and M. $m$. domesticus. Three talks focused on the multifaceted roles of this intriguing gene. Vaclav Gergelits (Institute of Molecular Genetics, Czech Republic) summarized his comprehensive analysis of non-crossover (NCO) gene conversion events in whole genome sequences from $\mathrm{PWD} / \mathrm{PhJ}$ chromosome substitution strains (CSSs) on the B6 background and progenitors, where NCO events are highly enriched. He found that B6 and PWD PRDM9 binding sites were often observed within 250 bases of NCO gene conversion events. Gene 
conversion events also overlapped with previously reported H3K4me3 modifications and DMC1 binding sites, providing additional evidence for evolutionary erosion of PRDM9 binding sites through NCO gene conversion. Chris Baker (The Jackson Laboratory) addressed the role of PRDM9 as a pioneer factor in male germ cells, specifying recombination hotspots by making chromatin accessible to other epigenetic modifiers. Baker identified one such candidate modifier that directly interacts with PRDM9 during the specification and repair of future double-strand break sites. Engineered mice lacking this enzyme recapitulate the loss of meiotic germ cells shown by $\operatorname{Prdm} 9$ knockout mice, demonstrating that this interacting partner of PRDM9 is essential for proper recombination hotspot activation. Tanmoy Bhattacharyya (The Jackson Laboratory) provided an overview of an intriguing potential role of a burst of TE insertions in modulating hybrid male sterility (HMS) between the PWD and B6 strains. Though the genetic interaction of $\operatorname{Prdm} 9$ and Chromosome (Chr) X in driving HMS is well characterized, Bhattacharyya presented work elucidating the mechanism of this interaction. B6 consomic mice hemizygous for Prdm9 and containing only a $50 \mathrm{Mb}$ fragment of the PWD Chr $\mathrm{X}$ phenocopied sterile (PWDxB6)F1 hybrids and displayed reduced recombination hotspot activation traditionally indicative of PRDM9 activity. Bhattacharyya also showed that the Llmd transposon is enriched on the PWD Chr X and bound directly by the B6 PRDM9 protein, providing the first evidence for a biochemical incompatibility between the B6 $\operatorname{Prdm} 9$ allele and the PWD Chr X.

David Beier (Seattle Children's Research Institute, USA) investigated a natural, evolutionary solution to the ubiquitous biological challenge of tissue repair. While most species undergo a pathogenic sequence of cellular events that lead to scar tissue formation at wound sites, African Spiny mice experience scarless tissue repair. Beier's group uncovered gene expression differences associated with scarless versus fibrotic kidney repair, indicating a genomic adaptation in African Spiny mice that confers their remarkable tissue regeneration properties.

Novel analytical approaches for querying massive scale datasets were also presented in this session. Leonard McMillan (UNC-Chapel Hill) noted that a major obstacle to analysis of high-throughput sequencing data is the technical expertise required to navigate multiple processing steps needed to extract biological insights. He outlined a computationally efficient and user-friendly pipeline for the exploration of sequencing data using the multi-string BurrowsWheeler transform. This pipeline allows the user to search for any k-mer in raw sequencing data and affords multiple instant advantages over more traditional exploratory analyses including: (i) local assembly of structural variation, (ii) variant discovery, and (iii) allele-specific gene expression analysis. In McMillan's words, this new pipeline allows scientists to fluidly "touch their [sequencing] data" and interrogate genome-scale data in a more interactive way.

Diverse mapping populations like the $\mathrm{CC}, \mathrm{DO}$, and heterogeneous stock (HS) impose a need for new statistical methods and software for QTL mapping. Notably, cryptic genetic structure, haplotype uncertainty, and imbalanced allele frequencies in these populations can lead to spurious associations. Greg Keele (The Jackson Laboratory) described a tool-miQTL—for remediating false positive findings. This program implements multiple imputation to stabilize QTL association scores and estimate their accuracy.

Anna Tyler (The Jackson Laboratory) developed a robust pipeline for candidate gene prioritization in quantitative genetic studies. Tyler's computational method for candidate prioritization was applied to a QTL for contextual fear learning in a cross between the B6-5XAD mouse model of Alzheimer's disease (AD) and BXD lines. First, mediation analysis was used to identify genes directly controlling the phenotype and derive measures of confidence of their involvement in the phenotype. Tyler then trained a support vector machine to classify genes within the QTL peak into each module. This method implicated Ythdf3 as a potential candidate regulator of contextual fear learning, even though this gene had never been linked to AD before. Overall, Tyler illustrated the power of her method to prioritize candidate genes in an unbiased manner.

Ongoing annotation of mouse genome features relies heavily on manual curation of gene function despite the relative difficulty of obtaining high-quality protein sequence compared to nucleic acids. In addition, genome features with restricted expression or atypical structure may elude detection or annotation. To improve this process, Jane Loveland and Toby Hunt (European Molecular Biology Laboratory, UK) developed phyloCSF, which infers protein function and identifies novel coding sequences by identifying regions of conservation under coding and non-coding evolutionary models. From this framework, Loveland and Hunt were able to add over 130 new protein coding genes to the mouse GENCODE database, demonstrating that phyloCSF is a promising new workflow for genome annotation.

\section{Animal models of human disease}

Multiple presenters reported new breakthroughs in human disease research enabled by diverse animal models of cancer, infection, immune-mediated disorders, and neurobehavioral pathologies.

Elizabeth Bryda (University of Missouri, USA) used a multi-species comparative approach to determine whether mutations identified in human disease patients are causal. Specifically, Bryda employed CRISPR-Cas9 to generate mouse models carrying mutations found in human renal 
cystic disease. She showed that knock-in mice harboring a human mutation recapitulated the human phenotype, indicating that the tested mutations are causal for disease.

Three talks focused on understanding how previously uncharacterized environmental factors such as the microbiome interact with the host genome and impact disease development. Dimitry Krementsov (University of Vermont, USA) investigated the interplay between the genome and microbiome in the context of the CNS autoimmune disease multiple sclerosis. Using 16s rRNA sequencing applied to a panel of consomic strains and microbiome transplant experiments, he identified complex bidirectional interactions between host genome and the gut microbiome influencing susceptibility to CNS autoimmunity. Jacob Moskowitz (University of Missouri) showed how host genetic divergence and the gut microbiota cooperatively contribute to intestinal tumor phenotype differences between divergent inbred $A p c^{M i n}$ colonies. Moskowitz used integrated genomic, gene expression, and metabolomics analyses to suggest a substantial role for bile acid metabolism in tumor initiation. James Amos-Landgraf (University of Missouri) presented research building off of previous results demonstrating that the complex intestinal microbiota impacts tumor burden in the Pirc rat model of colorectal cancer. New results implicated biofilm production from Desulfovibrio vulgaris, a sulfate-reducing microbe, as critical for tumor development.

Klaus Schughart (Helmholz Centre for Infection Research, Germany) presented work aimed at understanding how genetic variation shapes health outcomes following influenza virus infection. Schughart used CC mice to assess transcriptomic changes in the blood following infection and compared transcriptomic responses with data from prior human studies. He showed that gene expression responses are highly similar in humans and mice, arguing that mice provide a powerful in vivo model system for identification and validation of genes determining outcomes of influenza infection.

Two talks focused on mouse models mis-expressing specific oncogenes. Beverly Mock (National Institutes of Health, USA) presented a part of her extensive work on the transcription factor and potent oncogene MYC. Mock is using novel approaches to test MYC as a potential therapeutic target. She showed that a G4-quadruplex structure forms within the MYC promoter, which may be an actionable target for stabilization, and thus prevents its transcription. Lauren Tracey (Hospital for Sick Children) presented her work on the pluripotency regulator and oncogene PRDM14, which causes $\mathrm{T}$ cell leukemia when mis-expressed in blood cells. Using a proteomics strategy, she identified novel PRDM14 protein-protein interactions. When Tracey knocked out the strongest interactor, CBFA2T3, from the leukemia mouse model, mice no longer developed leukemia, implicating
CBFA2T3 as an important driver of PRDM14-induced leukemogenesis.

Several speakers highlighted the power of mouse models in studying complex neurological disorders. Monica Justice (Hospital for Sick Children) presented the results of her forward genetics suppressor screen in a mouse model of Rett Syndrome. This unbiased screen revealed a number of novel pathways that are disrupted in the Rett Syndrome model. Of note, Justice described an important role for tightly regulated DNA damage responses in the pathogenesis of Rett Syndrome for the first time. Using the same mouse model, Neeti Vashi (Hospital for Sick Children) presented her characterization of respiratory symptoms that appear to be caused by increased lung lipids. In Rett Syndrome, respiratory illness is a major contributor to patient death but is rarely studied in the field. Vashi's molecular dissection of phenotypic etiology uncovered separate neurological and lung-specific contributors to the disease. Importantly, she showed that respiratory symptoms can be ameliorated with lipid-lowering drugs. Yann Herault (Institute of Genetics and Molecular and Cellular Biology, France) presented his work on understanding the genetic causes of intellectual disability. Herault developed a panel of mouse models harboring mutations found in human intellectual disability cases. These models were then subjected to a series of standardized behavioral screens, and selected mouse models were treated with pharmacological agents to evaluate their therapeutic potential to reverse behavioral phenotypes. Overall, Herault showed that mice broadly recapitulated behavioral features and cognitive deficits associated with intellectual disability in humans.

Understanding the sources of genetic and environmental susceptibility to congenital malformations is critical to developing treatments and preventative measures. Sally Dunwoodie (Victor Chang Cardiac Research Institute, Australia) presented her lab's work dissecting two genes recently implicated in family studies of congenital heart and vertebral defects, Наaо and Куnи. Dunwoodie's group developed mice with null mutations in both genes and found that these mice had reduced nicotinamide adenine dinuclelotide (NAD) levels and increased precursors of this molecule but, surprisingly, displayed no phenotype. However, upon the removal of vitamin B3 from the diet, these mice phenocopied the human patients carrying these null alleles. Supplementation with vitamin B3 rescued these phenotypes, demonstrating that both environmental and genetic modification of NAD deficiency is critical to proper embryogenesis in both mice and humans.

Gray coat color mutants in mice represent an exciting model of pheomelanin regulation in mammals, especially given the genetic heterogeneity underlying this phenotype. Dawn Watkins-Chow (National Institutes of Health) presented a detailed analysis of two different gray coat color mutants: grizzled $(g r)$ and grey intense (gri). Through 
genome-wide association, Watkins-Chow linked a deleterious mutation within the $M f_{s} d 12$ gene to variation in skin pigmentation among African populations. She also demonstrated widely pleiotropic effects of a Usp32 mutation within the gri locus on viability, bone density, and tooth morphology. Extension of this work into fixed genetic backgrounds and evaluating the mode of action of each mutation will be a promising route to identify the mechanistic connections between pheomelanin mutants and other diseases, including skin cancer.

CRISPR-Cas9 has become the gold standard for gene editing in molecular biology, enabling facile construction of animal models of human disease. Lydia Teboul (MRC Harwell Institute, UK) provided a reminder that, despite the power of CRISPR approaches, the technique is not always as straightforward as it seems. Teboul demonstrated that targeted deletions can lead to high variability in outcomes outside of the predicted sequence, including larger deletions and rearrangements. Her work emphasized the need to screen G0 founders in depth to discern the range of mutants generated after CRISPR-mediated genome editing. Staggered Sanger sequencing or long-read sequencing is necessary to capture the potential complexity of unexpected alterations.

\section{Translational and systems genetics}

Despite transformative advances from powerful animal models, our knowledge of the genetic basis of many human diseases is incomplete. This knowledge gap poses a substantial barrier to the characterization and ultimate development of novel treatment strategies. IMGC members are pioneering new approaches to identify genetic loci with mechanistic contributions to disease.

Several investigators demonstrated that highly genetically diverse inbred strains such as the Collaborative Cross vastly improve our mapping capabilities, allowing for increased resolution when identifying loci associated with phenotypes of interest in a systems genetics approach. Elena Mogilyanksy (Thomas Jefferson University, USA) sought to elucidate new genetic modifiers of the $A p c^{M i n}$ phenotype by establishing $A p c^{M i n}$ crosses with different CC strains. Through evaluation of the intestinal tumor phenotype of F1 hybrids, Mogilyansky aims to map QTLs associated with distinct tumor burdens and thus identify candidate genes with a role in tumor development. Samir Kelada's (UNC-Chapel Hill) talk focused on identifying genetic control of mucin expression in acute respiratory responses to foreign material. Using recombinant inbred CC lines, Kelada identified BpifBI as a candidate in the control of MUC5 expression and further displayed exciting new data showing that loss of BpifBI does in fact control host response by altering MUC5 expression. Kelsey Noll and Lisa Tarantino (UNC-Chapel Hill) used similar systems genetics approaches to map genes associated with phenotypes of interest in the CC. Noll traced interindividual variability in susceptibility to influenza to a QTL the NLRP1 inflammasome locus, which significantly correlated with the IgG immune response. Tarantino assessed variable locomotor responses to cocaine exposure in mice as a basis to identify candidate genes responsible for substance abuse disorders. She then used QTL and eQTL mapping data to identify $C h s t 8$ as a priority candidate for changes in addiction-related behaviors.

Paola Giusti-Rodriguez (UNC-Chapel Hill) utilized CC recombinant inbred cross (RIX) mice as a model system to address whether antipsychotic phenotypes were heritable and responsive to haloperidol treatment. She found that chronic exposure to haloperidol reduced open field activity and modified orofacial phenotypes, while acute exposure increased the amount of time spent on an inclined screen. She observed considerable genetic variance for these traits, as well as appreciable gene-by-treatment effects, demonstrating that this system could be utilized in the future to characterize the biology underlying unpredictable responses to haloperidol observed in the human population. Determining the responses of populations at risk of various environmental exposures requires reproducible, genetically heterogeneous populations. Danila Cuomo (Texas A\&M University) also utilized CC RIX mice to assess the effects of early-life lead exposure. She observed broad variation in blood lead levels and blood cell counts across CC RIX hybrids, as well as robust epigenetic alterations after exposure. This work is an important first step toward identifying biomarkers of lead exposure among genetically diverse individuals from human populations.

John Shorter (UNC-Chapel Hill) characterized phenotypes related to epilepsy in the $\mathrm{CC}$, demonstrating that seizures, kindling, and sudden unexplained death are heritable across the panel and controlled by distinct genetic loci. Using F2 crosses between CC strains, Shorter mapped several QTLs for these traits. Alexandra Schafer (UNC-Chapel Hill) characterized the effects of Ebola virus (EBOV) infection among $\mathrm{CC}$ strains, for which there was substantial variation. In an F2 cross between two CC lines, she demonstrated that symptoms of EBOV infection, like mortality and weight loss, have a distinct genetic architecture from the pathological signatures of EBOV infection in the liver. This quantitative genetic approach is powerful for understanding the genetic basis of various responses to infection.

Other investigators utilized diversity among classical inbred mouse strains to gain novel genetic insights into disease-relevant phenotypes. Anna Salvador (Texas A\&M University) discussed the underlying genetics that control adipogenesis and weight-gain responses with exposure to high- and low-carbohydrate diets in combination with a high-fat diet. Salvador found differential responses 
between B6 and FVB inbred strains and exploited this observation to map candidate genes such as Klf3, which has a previously characterized role in adipogenesis. Identification of gene signatures associated with responses to different diets will ideally enable more personalized dietary recommendations. Lisa Gralinski (UNC-Chapel Hill) investigated the genetic basis of response to SARS-CoV infection by leveraging differences in mortality between two extremely closely related mouse strains. This reduced complexity cross allowed her to identify and finely map QTLs underlying mortality by interrogating private SNPs to each strain. David Aylor (North Carolina State University) screened a collection of male classical inbred strains and CC strains for reproductive defects associated with exposure to diethylstilbestrol (DES), a potent synthetic estrogen. While some strains appear relatively insensitive, many strains are sterile after exposure to DES and exhibit testicular germ cell loss. Surprisingly, there were substantial differences in Leydig and Sertoli cell populations during the establishment of germ cells within sensitive strains. These results present an interesting case where gene-by-environment interactions are driven by heritable differences in phenotypic variation that are amplified by toxic exposure in early development.

Protein-coding mutations present a classical genetic paradigm for human disease. Fernando Benavides (MD Anderson Cancer Research Center, USA) previously demonstrated that the ebouriffé mutation, which causes male and female infertility, is a premature stop codon that truncates the structural domain of LRCC $8 \mathrm{~A}$. This gene is essential for proper volume-regulated anion channel (VRAC) activity and, using various engineered mice, Benavides demonstrated that Lrcc8a-dependent VRAC activity is an essential component of germ cell development and spermiogenesis.

Emerging genomic technologies are empowering the functional discovery and roles of regulatory variants in the pathogenesis of human disease. Sarah McClymont (Johns Hopkins University, USA) employed ATAC-seq in dopaminergic neurons to identify regions of open chromatin in a mouse model of Parkinson's Disease. McClymont identified a midbrain-specific ATAC-seq peak that may be contributing the midbrain specific expression of SNCA, the protein that aggregates in Parkinson's disease neurons. Further, she presented her discovery of two variants within this enhancer that are significantly associated with Parkinson's disease. William Law (Johns Hopkins University) used a modified STARR-seq pipeline to identify and functionally assay putative regulatory elements. He selected over 3000 GWASassociated SNPs implicated in human neurological disease and assayed them for regulatory activity with this technique. Over 300 putative enhancers were identified in his screen for follow-up to determine the target genes they regulate.

\section{Resources and technical advances}

Continued advancement in the fields of mammalian genetics and genomics requires accessible resources that facilitate global data transfer throughout the community. Several talks at IMGC2018 highlighted the meeting as an important platform for updating the community on the additions of new, and updates to current resources.

Carol Bult (The Jackson Laboratory) provided an overview of several key improvements to the Mouse Genome Informatics (MGI) database implemented by The Jackson Laboratory. Catering to a growing interest in cataloguing functional variation among inbred mouse strains, MGI has now implemented a number of features that facilitate easy navigation of newly generated reference genomes for many popular mouse strains. Included in these features is the strain comparison, which enables the user to select strains to compare and directly query popular repositories for variants and sequences. Adam Frankish (European Bioinformatics Institute, UK) relayed the efforts of the GENCODE consortium to produce reference-quality annotations of protein-coding genes, pseudogenes, long non-coding RNAs, and small RNAs in the mouse. Frankish emphasized that, despite good gene coverage in GENCODE data sets, a continued challenge will be identifying alternative transcripts in mice. Thus, new transcript models are being incorporated based on emerging transcriptomics data to fill these gaps. Jennifer Smith (Medical College of Wisconsin, USA) delivered updates on the Rat Genome Database (RGD), focusing on the PhenoMiner tool which provides a breadth of quantitative phenotype measurements for reference in the rat. Notably, this resource enables the community to more easily select appropriate models to address specific questions based on a strain's reference ranges for phenotypes of interest. Danny Arends (Humboldt-Universität zu Berlin, Germany) outlined the newest improvements to the BXD recombinant inbred panel, which has provided over 191 independent strains that have been phenotyped for thousands of traits. After integrating over 4000 additional markers to the genetic map, the power and resolution for QTL mapping have been dramatically increased. These resources have been integrated with $\mathrm{R} / \mathrm{qt} 2$, gene ontology analysis pipelines, gene expression network analysis algorithms, and PheWAS.

The International Mouse Phenotyping Consortium (IMPC), which seeks to generate and phenotype a mouse knock-out for every gene, has completed 6000 genes and an additional 14,000 are scheduled. Valérie GailusDurner and Martin Hrabě de Angelis (Helmholz Zentrum Munich, Germany) presented both updates and examples of the power of this approach. Gailus-Durner found that the Uqcrh KO mouse mimicked phenotypes of a rare 
human mitochondrial respiratory chain disease. Upon further study, she identified novel phenotypes in the mice that are now being monitored in patients, demonstrating how mouse models are particularly powerful for rare diseases where only small numbers of human patients exist for study. Hrabě de Angelis described how he harnessed the strengths of the IMPC to study complex metabolic diseases such as obesity and diabetes. He performed largescale metabolic phenotyping on over 2000 mouse knockout lines. This high-throughput strategy has launched new efforts to elucidate the network of interacting genes involved in metabolism.

Thomas Keane (European Bioinformatics Institute) presented his on-going work expanding the evolutionary scope genome resources for the rodents. Giving special emphasis to species in the genus Acomys, Keane introduced scaffoldlevel genomes produced with $10 \mathrm{X}$ Chromium sequencing. Dedicated annotation effort is being devoted to these genomes with the promise of revealing mechanisms of the unique biology of diverse rodent taxa.

Finally, Karen Svenson (The Jackson Laboratory) presented valuable updates on her studies addressing animal welfare considerations, including recommendations for housing density of mice. Svenson determined that trio breeding provides increased litter size and survival, demonstrating that breeding arrangements can have an important impact on animal welfare. Studies addressing these welfare issues provide essential data for the community to ensure the continued practice of ethical animal research.

\section{Terry Magnuson-Verne Chapman lecture}

The Verne Chapman Memorial Lecture, established in 1997, is awarded annually to a scientist who exemplifies the qualities of the award namesake, IMGS co-founder Dr. Verne M. Chapman. Dr. Chapman's legacy is one of the generous mentors and a leader in the scientific community. This year, Terry Magnuson (Vice Chancellor of Research at UNCChapel Hill) was awarded this prestigious lecture.

The lecture summarized Magnuson's pioneering work on chromatin-remodeling complex dynamics and their role in development and disease. Magnuson first focused on the SWI/SNF complexes, of which thousands of forms exist. These ATP-dependent remodeling complexes are enriched at regions of open chromatin, namely putative enhancers and promoters, and consist of 3-17 protein subunits. One component of these complexes, the ARID family of proteins, is the most commonly mutated chromatin regulators in human cancers. Magnuson detailed his lab's work elucidating the genome-wide dynamics of these complexes and showed that different ARIDs that co-localize at the same genes can work cooperatively or competitively with each other. In a site- and context-dependent manner, the presence of specific ARID subunits can promote or repress gene expression. To highlight the importance of this, Magnuson described the high occurrence of ARID1A loss of function mutations co-occurring with PI3KCA activation mutations in ovarian cancer, which together promote cell cycle progression and proliferation. Magnuson and his team further confirmed this finding in an in vivo mouse model.

Magnuson also presented exciting new work that led to the identification of canonical and non-canonical targets of the human INO80 chromatin remodeling complex. Noncanonical targets are enriched for the repressive PRC2 complex and H3K27me3 marks, and are only associated with the INO80 ATPase subunit. Conversely, canonical targets are marked with $\mathrm{H} 3 \mathrm{~K} 27 \mathrm{ac}$ and open chromatin and are associated with all INO80 complex modules. Finally, Magnuson demonstrated that differences in the composition of remodeling complexes has critical roles during development and disease states. This level of complexity affords remarkable precision in gene regulation and may also help explain complex disease phenotypes.

\section{Beth L Dumont-Mary Lyon awardee lecture}

In 2018, the IMGS established a new award to honor one of its founding members, Dr. Mary Frances Lyon. Dr. Lyon was known for her groundbreaking work describing random $\mathrm{X}$-chromosome inactivation for the first time, as well as her pivotal contributions to the field of mouse genetics. Dr. Lyon began her career at a time when few women became scientists, and she served as a powerful advocate and role model for junior female scientists throughout her lifetime. In light of her contributions, the Mary Lyon Award lecture is given to a female junior investigator at the faculty level. The inaugural award was presented to Beth Dumont (The Jackson Laboratory) at IMGC 2018. Dumont's seminar was entitled "Sex chromosome heterochromatin and the emergence of asynaptic sex chromosomes in North American voles".

Dumont introduced the pseudoautosomal region (PAR), the narrow region of homology between the sex chromosomes that is critical for the proper segregation of the $\mathrm{X}$ and $\mathrm{Y}$ in most mammals. Intriguingly, this region is one of the fastest evolving loci in the mammalian genome. Dumont described how her lab is gaining mechanistic insight into the function of this region by conducting comparative analyses across different rodent species. As an example of this approach, she described recent work utilizing the natural variation in meiotic sex chromosome programs in North American voles. Using a single chromosome sequencing strategy, Dumont characterized sex chromosome architecture and $\mathrm{X} / \mathrm{Y}$ homology in three vole taxa with distinct modes of sex chromosome segregation. Her work uncovered an 
intriguing link between the accumulation of heterochromatic sequences on the sex chromosomes and non-canonical, PARindependent mechanisms of sex chromosome segregation.

\section{Forward genetics panel}

The Forward Genetics Panel session was hosted by INFRAFRONTIER. Nobel laureate Bruce Beutler began the session with his seminar "Mutagenesis with real-time cause and effect." Beutler described the foundation of forward genetics screens, in which a mutagen, commonly ENU in mice, is used to create random germline mutations throughout the genome. Changes in phenotypes of the progeny are then traced back to the causative mutation. In this way, novel functions for genes can be identified, including roles in alternative pathways or modification of disease states. The Beutler Lab has undertaken an ENU forward genetics screen in mice, and boasts an impressive phenotyping pipeline where they examine immunological, neurological, and metabolic phenotypes, to name a few. To date, they have examined over 150,000 coding or splicing changes in over 20,000 mouse genes. A newly developed machine learning algorithm helps to better predict candidate gene mutations, which now has an $80 \%$ validation success rate. Knock-out mice are subsequently generated using CRISPR-Cas9 to confirm candidate genes and conduct mechanistic studies. To highlight the power of this approach, Beutler described how his team identified 85 mutations in 39 genes in the TLR signaling pathway. Sixteen of these genes were completely novel and have improved our understanding of this important pathway.

A panel discussion followed on the power of forward genetics, chaired by Martin Hrabě de Angelis, with pioneers in the field: Ruth Arkell, David Beier, Bruce Beutler, Monica Justice, Laura Reinholdt and Nadia Rosenthal. All of the speakers reinforced the value of mouse models and the importance of conducting studies with a thorough knowledge of mouse genetics to improve reproducibility and reliability. The panel addressed issues such as how to break through funding barriers and better highlight the importance of mouse models for understanding basic biology as well as human disease.

\section{Conclusions and looking ahead}

As was evident throughout IMGC2018, the rapidly evolving landscape of genetics and genomics has paved the way for exciting new approaches to a myriad of challenges facing today's researchers. Increased efficiency in genetic manipulation techniques, new systems genetics approaches, and expanded data sharing resources have substantially improved our ability to locate and validate important genes in health and disease. Furthermore, significant advances in singlecell resolution of the transcriptome, new computational approaches, and growing interest in how environmental factors influence genetics provided new insight into the complex factors that drive both developmental and disease phenotypes. The availability of these technologies in mammalian research models exemplifies a critical role for these systems in our continued forward progress.

The IMGS eagerly anticipates the 2019 meeting, which will be held September 25-28 in Strasbourg, France. In 2020, the IMGS will once again hold its meeting as part of The Allied Genetics Conference, April 22-26 in Washington D.C, where geneticists representing a variety of major model systems will come together to share new progress (Bloom et al. 2017). Information and updates regarding upcoming meetings can be found at www.imgs.org and www.confe rences.genetics-gsa.org.

Acknowledgements We thank Darla Miller and Linda Siracusa for thoughtful comments on a draft version of this manuscript.

\section{Compliance with ethical standards}

Conflict of interest The authors declare that they have no conflict ofinterest.

\section{References}

Bloom JC, Tran TN, Yamulla RJ (2017) Mouse genetics 2016: meeting report. Mamm Genome 28(3-4):81-89

Sanchez-Andrade G, Moskowitz J, Howe S, Gunn TM (2018) Meeting report: 31st international mammalian genome conference, mammalian genetics and genomics-from molecular mechanisms to translational applications. Mamm Genome 29(5-6):299-309

Publisher's Note Springer Nature remains neutral with regard to jurisdictional claims in published maps and institutional affiliations. 